\title{
A Rare Case of Salmonella paratyphi-A Pleural Effusion
}

\author{
V.P. Bansal, Tazammul Hussain* and M.V. Mulay \\ MGM Medical College, Auranagabad, Maharashtra, India \\ *Corresponding author
}

\section{A B S T R A C T}

\section{Keywords \\ Salmonella \\ paratyphi, \\ Orthopnoea, \\ Pleural effusion \\ and Pleural fluid \\ Article Info \\ Accepted: \\ 12 April 2019 \\ Available Online: \\ 10 May 2019}

\section{Introduction}

The Salmonelleae contain a single genus, Salmonella, named after American microbiologist, D.E.Salmon. (1). The growth of serotypes Salmonella typhi and Salmonella paratyphi is restricted to human hosts, causes enteric fever. More than 200 serotypes of Salmonella are pathogenic to humans, in whom they often cause gastroenteritis and can be associated with localized infections and/or bacteremia. (2).Salmonella spp are known to be a rare respiratory tract pathogen (3). To the best of our knowledge, until now, one case has been reported of pleural effusion due to
Salmonella paratyphi A, in India (4). Being a rare isolate, at unusual site, in an immunocompetent individual, this case is reported.

\section{Case report}

A $22 \mathrm{yr}$ old girl presented with history of breathlessness on exertion from past 3 month, increased gradually. Patient presented with history of orthopnoea, there was history of dry cough since eight days, fever on \& off, not associated with chills, since eight days, abdominal pain, mostly after eating food and also history of passing loose stools since once 
week, increased frequency. On physical examination she had temperature of $38^{\circ} \mathrm{C}$. The respiratory rate was 26 per minute. Decreased respiratory movement on right side. Trails sign was positive towards left.; Breath sounds were absent in the right hemithorax. Investigation revealed total leucocyte count $26000 / \mathrm{mm}^{3}$. SGOT(aspartate aminotransferase) $21 \mathrm{IU} / \mathrm{L}$, SGPT (alanine aminotransferase) 37 IU/L, Alkaline phosphatase 188U/L. Pleural tapping was done on two consecutive days. Pleural fluid (First tapping) Protein $4.3 \mathrm{gm} / \mathrm{dl}$ Sugar <20 $\mathrm{mg} / \mathrm{dl}$ Pleural fluid was sent for culture (sample1) Pleural fluid (Second tapping) Protein $2.3 \mathrm{gm} / \mathrm{dl}$ Sugar <20 mg/dl Pleural fluid sent for culture (sample2)

Gram stain of pleural fluid revealed many polymorphonuclear leucocytes with gram negative bacilli. Pleural fluid Culture: (of two consecutive samples - pleural fluid, collected on consecutive days) On 5\% sheep Blood agar: greyish white, opaque, circular, elevated, non hemolytic colony. On MacConkey's agar: non lactose fermenting colonies. Catalase positive, Oxidase negative. Gram stain of colony - short, gram negative bacilli. Pleural fluid TB-PCR was negative. By using Vitek2 Compact System (bioMérieux), Gram negative card - the isolate was identified as Salmonella ser. Paratyphi A, which was sensitive to Ampicillin, Amoxicillin/clavulanic acid, Imipenem, Meropenem, Amikacin, Gentamicin, Tigecyline, Cotrimoxazole, \& SDD-Cefepime, and resistant to Ceftriaxone, Ciprofloxacin.

The isolate was later confirmed with manual biochemical tests as well as serological test which was positive agglutination with Salmonella antisera polyvalent $\mathrm{O}$ and Salmonella O2 factor, suggestive of Salmonella paratyphi A. On admission, patient treatment was started with
Ceftriaxone. Once culture report was available the treatment was changed to amikacin. Treatment follow up could not be done as patient requested for early discharge.

The tribe salmonelleae contain only one genus, Salmonella, named after American microbiologist, D.E. Salmon.(1). Salmonella (all serotypes) are motile, gram-negative rods commonly isolated human and animal intestine.(2).Salmonella are worldwide, nonspore forming, Gram negative, facultative anaerogenic, motile bacilli of the Enterobacteriaceae family. Extraintestinal complications of salmonella are rare. Globally there are few Salmonella paratyphi A cases reported. (3) Until now previously only one case of pleural effusion due to Salmonella paratyphi-A has been reported in the year 2002 by $\mathrm{P}$ mathur in an immunocompetent pediatric patient, which was sensitive to Ciprofloxacin and Ceftriaxone and resistant to Amoxicillin, Cotrimoxazole.

In the present study, we isolated the Salmonella paratyphi A from pleural fluid which was sensitive to amoxicillin and Cotrimoxazole \& resistant to ciprofloxacin and ceftriaxone. (4). We have performed TB PCR which was negative, thereby ruling out tuberculosis being the cause of pleural effusion in our patient. Previously, Salmonella paratyphi A has been reported from cases of Liver cyst, endocarditis, necrotising fasciitis, spleenic abscess, breast abscess, ovarian abscess, osteomyelitis, brain abscess, meningitis, empyema, pericarditis. (8), (9), (11), (7). Salmonella paratyphi A in pleural fluid bis a rare presentation, hence this case is reported.

In conclusion, in a case of pleural effusion, ruling out tuberculosis by TB PCR and repeat isolation of Salmonella paratyphi $\mathrm{A}$ in consecutive sample confirms the rare presentation of Salmonella paratyphi A. 


\section{References}

1. Koneman's Color Atlas And Textbook Of Diagnostic Microbiology $6^{\text {th }} \mathrm{Ed}$, Ch 6, Pp. 251.

2. Bailey and Scott, diagnostic microbiology 14 th ed.

3. Harrison principles of internal medicine $19^{\text {th }}$ ed Vol. 2, ch 190, Pp 1049.

4. P Mathur Indian pediatric editorial 2003; 40: 252-254.

5. Pradeep Kumar Eur Spine J (2008) 17:754-755.

6. A.Sangwaiya, Journal of Tropical Medicine, Volume 2009; Article ID 456810, 3 Pp.
7. Dar et al., Salmonella paratyphi A endocarditis complicating chronic rheumatic heart disease, Nigerian Journal of Cardiology. July - December 2014. Vol. 11, Issue 2.

8. Sudhaharan et al., - Extra-intestinal salmonellosis, J Infect Dev Ctries 2014; 8(7): 831-837.

9. Smita Sood, Salmonella Breast Abscess; Journal of Clinical and Diagnostic Research. 2015 Sep, Vol-9(9): DD03DD04.

10. Saeed - Salmonella pneumonia with encysted empyema Infect Dev Ctries 2016; 10(4): 437-444.

11. Benu Dhawan, Intractable \& Rare Diseases Research. 2018; 7(2):130-133.

\section{How to cite this article:}

Bansal, V.P., Tazammul Hussain and Mulay, M.V. 2019. A Rare Case of Salmonella paratyphi-A Pleural Effusion. Int.J.Curr.Microbiol.App.Sci. 8(05): 1344-1346.

doi: https://doi.org/10.20546/ijcmas.2019.805.153 